\title{
Controllable Continuous evolution of electronic states in a single quantum ring
}

\author{
Tapash Chakraborty ${ }^{1}$, Aram Manaselyan $^{2}$, Manuk Barseghyan ${ }^{2}$ and David Laroze ${ }^{3,4}$ \\ ${ }^{1}$ Department of Physics and Astronomy, University of Manitoba, Winnipeg, Canada R3T 2N2 \\ ${ }^{2}$ Department of Solid State Physics, Yerevan State University, Yerevan, Armenia \\ ${ }^{3}$ Instituto de Alta Investigación, Universidad de Tarapacá, Casilla 7D, Arica, Chile \\ ${ }^{4}$ Yachay Tech University, School of Physical Sciences and Nanotechnology, 00119-Urcuquí, Ecuador and \\ E-mail: Tapash.Chakraborty@umanitoba.ca, amanasel@ysu.am, mbarsegh@ysu.am,dlarozen@uta.cl
}

\begin{abstract}
Intense terahertz laser field is shown to have a profound effect on the electronic and optical properties of quantum rings, where the isotropic and anisotropic quantum rings can now be treated on equal footing. We have demonstrated that in isotropic quantum rings the laser field creates irregular $\mathrm{AB}$ oscillations that are usually expected in anisotropic rings. Further, we have shown for the first time that intense laser fields can restore the isotropic physical properties in anisotropic quantum rings. In principle, all types of anisotropies (structural, effective masses, defects, etc.) can evolve as in isotropic rings, in our present approach. Most importantly, we have found a continuous evolution of the energy spectra and intraband optical characteristics of structurally anisotropic quantum rings to those of isotropic rings, in a controlled manner, with the help of a laser field.
\end{abstract}

Research on the electronic and optical properties of quantum confined nanoscale structures, such as quantum dots and quantum rings has made great strides in recent years in unraveling new phenomena and their enormous potentials in device applications. In this context, quantum rings with its doubly-connected structure attracts special attention. Its unique topolocal structure provides a rich variety of fascinating physical phenomena in this system. Observation of the Aharonov-Bohm (AB) oscillations [1] and the persistent current [2] in small semiconductor quantum rings (QR), and recent experimental realization of QRs with only a few electrons 3, 4 have made QRs an attractive topic of experimental and theoretical studies for various quantum effects in these quasione-dimensional systems [5]. In particular, recent work has indicated the great potentials of QRs as basis elements for a broad spectrum of applications, starting with terahertz detectors [6], efficient solar cells 7] and memory devices [8], through electrically tunable optical valves and single photon emitters [9, 10]. We have also worked previously on QRs in new materials such as graphene systems [11] and $\mathrm{ZnO}$ [12] with interesting outcomes reported in [13] and [14] respectively.

Although almost circular or slightly oval shaped QRs have been fabricated by various experimental groups $15-$ [18], anisotropic QRs are the ones most commonly obtained during the growth process [17, 19 21]. Theoretically the effect of anisotropy on electronic, magnetic and optical properties of quantum rings have been investigated by various authors $22-26]$. In those studies, different types of anistropies were explored. For example, in Ref. [22, 24, 25] the shape anisotropy of the QR was considered, while in Ref. 23] the anisotropy associated with defects was studied, and in 26] the effective mass anisotropy was investigated. In all these cases it was shown that the anisotropy can dramatically alter the $\mathrm{AB}$ oscillations in the QR. In particular, in Ref. [26] it was demonstrated that the unusual $\mathrm{AB}$ oscillations caused by the effective mass anisotropy in the QR can be converted to usual $\mathrm{AB}$ oscillations if the $\mathrm{QR}$ has an elliptical shape. However, in order to experimentally confirm these results, one needs to grow QRs with different anisotropies and compare their measurable optical and magnetic characteristics individually.

Here we consider the effect of a terahertz intense laser field (ILF) on isotropic and anisotropic QRs and demonstrate that in the case of isotropic QRs the ILF can create irregular $\mathrm{AB}$ oscillations that are characteristics of anisotropic rings. Additionally, we have shown for the first time that in case of anisotropic QRs the ILF can be used as an anisotropy controlling tool with the help of which it will be possible to visualize both the isotropic and anisotropic properties on a single QR. For example, we have shown that the irregular $\mathrm{AB}$ oscillations obtained for the elliptic QR can be made regular with the help of the ILF. Therefore the ILF can unify all the electronic properties of isotropic and anisotropic rings in a single system.

Our system consists of a two-dimensional anisotropic QR structure containing electrons that are under the action of laser radiation and an external magnetic field that is oriented along the growth direction. The laser field is represented by a monochromatic plane wave of frequency $\omega$. The laser beam is non-resonant with the semiconductor structure, and linearly polarized along a radial direction of the structure (chosen along the $x$ - axis). In the non-interacting case, the electron motion is described by the solution of the time dependent Schrödinger equation

$$
\begin{aligned}
{\left[\frac{1}{2 m}\left(\widehat{\mathbf{p}}-\frac{e}{c}\left(\mathbf{A}(t)+\mathbf{A}_{m}\right)\right)^{2}+\right.} & V(x, y)] \Phi(x, y, t) \\
& =i \hbar \frac{\partial}{\partial t} \Phi(x, y, t),
\end{aligned}
$$

where $m$ is the electron effective mass, $\mathbf{p}$ is the lateral momentum of the electron, $e$ is the absolute value of the electron charge, $\mathbf{A}(t)=\mathbf{e}_{x} A_{0} \cos (\omega t)$ is the laser field's vector potential, where $\mathbf{e}_{x}$ denotes the unit vector on the $x$-axis. In Eq. (1), $\mathbf{A}_{m}$ is the vector potential of the magnetic field which is chosen to be $\mathbf{A}_{m}=(0, B x, 0)$. In this case the scalar product $\left(\mathbf{A}(t) \cdot \mathbf{A}_{m}\right)=0$. 
For the lateral confinement potential $V(x, y)$ we have chosen the model of finite, square-well type, which can be written as:

$$
V(x, y)= \begin{cases}0, & \text { if } R_{1} \leq \sqrt{x^{2}+\left(y / \sqrt{1-\varepsilon^{2}}\right)^{2}} \leq R_{2} \\ V_{0}, & \text { otherwise }\end{cases}
$$

where $R_{1}$ and $R_{2}$ are the inner and outer radii of the $\mathrm{QR}$ respectively, the $\varepsilon$ describes the anisotropy of the QR $(\varepsilon=0$ corresponds to the case of circular QR) 26].

Using the dipole approximation and KramersHennerberger unitary transformation 27] in the highfrequency regime 28 32] the laser-dressed energies of the QR can be obtained from the following time-independent Schrödinger equation

$$
\begin{array}{r}
{\left[\frac{1}{2 m}\left(\widehat{\mathbf{p}}-\frac{e}{c} \mathbf{A}_{m}\right)^{2}+V_{d}(x, y)\right] \Phi_{d}(x, y)} \\
=E_{d} \Phi_{d}(x, y),
\end{array}
$$

where $V_{d}(x, y)$ is the time-averaged laser-dressed potential that can be expressed by the following analytical expression [33, 34]

$$
\begin{aligned}
V_{d}(x, y) & =\frac{V_{0}}{\pi} \operatorname{Re}\left[\pi-\theta\left(\alpha_{0}-x-\Gamma_{1}\right) \arccos \left(\frac{\Gamma_{1}+x}{\alpha_{0}}\right)+\right. \\
& +\theta\left(\alpha_{0}-x-\Gamma_{2}\right) \arccos \left(\frac{\Gamma_{2}+x}{\alpha_{0}}\right)- \\
& -\theta\left(\alpha_{0}+x-\Gamma_{1}\right) \arccos \left(\frac{\Gamma_{1}-x}{\alpha_{0}}\right)+ \\
& \left.+\theta\left(\alpha_{0}+x-\Gamma_{2}\right) \arccos \left(\frac{\Gamma_{2}-x}{\alpha_{0}}\right)\right],
\end{aligned}
$$

where $\theta(u)$ is the Heaviside unit-step function and $\Gamma_{i}=\operatorname{Re}\left(\sqrt{R_{i}^{2}-\left(y / \sqrt{1-\varepsilon^{2}}\right)^{2}}\right)$. The parameter $\alpha_{0}=$ $\left(e / m \epsilon_{h}^{1 / 4} \nu^{2}\right) \sqrt{I /\left(2 c \pi^{3}\right)}$ describes the strength of the laser field, and comprises both the intensity $I$ and frequency $\nu$ of the laser field that can be chosen for a broad range in units of $\mathrm{KW} / \mathrm{cm}^{2}$ and $\mathrm{THz}$ correspondingly [32]. $\epsilon_{h}$ is the high-frequency dielectric constant of the system. In Fig. 1 the schematic picture of dressed potential for different values of the ILF parameter $\alpha_{0}$ is presented. The circular and elliptic cases of QRs are shown. The laser dressed energy eigenvalues $E_{d}$ and eigenfunctions $\Phi_{d}(x, y)$ may be obtained by solving Eq. (3) with the help of the exact diagonalization technique. The eigenfunctions are presented as a linear expansion of the eigenfunctions of the two-dimensional rectangular infinitely high potential well [33, 34].

We have also considered here the intraband optical transitions in the conduction band. According to the Fermi golden rule for the $x$-polarization of the incident light the intensity of absorption in the dipole approximation is proportional to the square of the matrix element $M_{f i}=\langle f|x| i\rangle$, when the transition goes from the initial

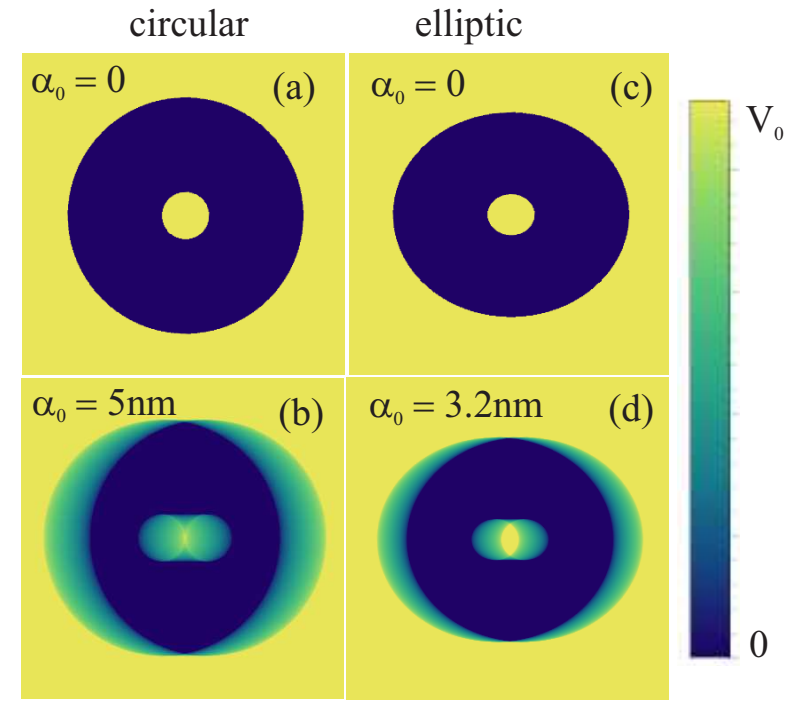

FIG. 1: The density plot of the dressed confinement potential for different values of the ILF parameter $\alpha_{0}$ for both circular and elliptic QRs.

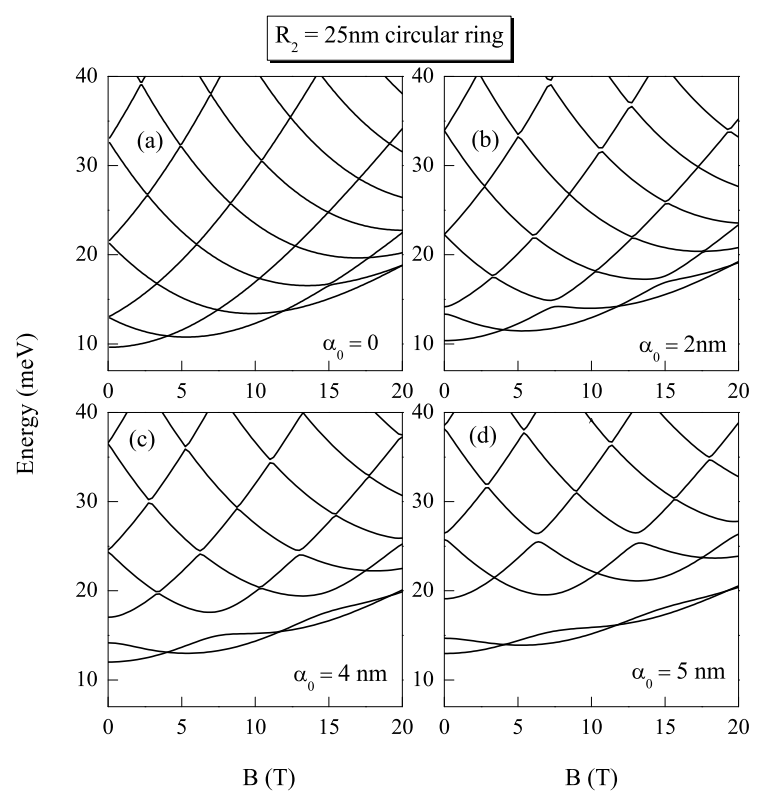

FIG. 2: The low-lying energy levels of a circular QR as a function of the magnetic field $B$ for different values of the laser field parameter $\alpha_{0}$. The results are for $R_{2}=25 \mathrm{~nm}$.

state $|i\rangle$ to the final state $|f\rangle$. In this work we always consider $|i\rangle$ to be the ground state.

Our numerical studies are carried out for GaAs QRs having parameters $V_{0}=228 \mathrm{meV}, m=0.067 m_{0}\left(m_{0}\right.$ is

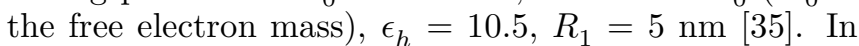
Fig. 2, the low-lying energy levels of circular QR with outer radius $R_{2}=25 \mathrm{~nm}$ are presented as a function 


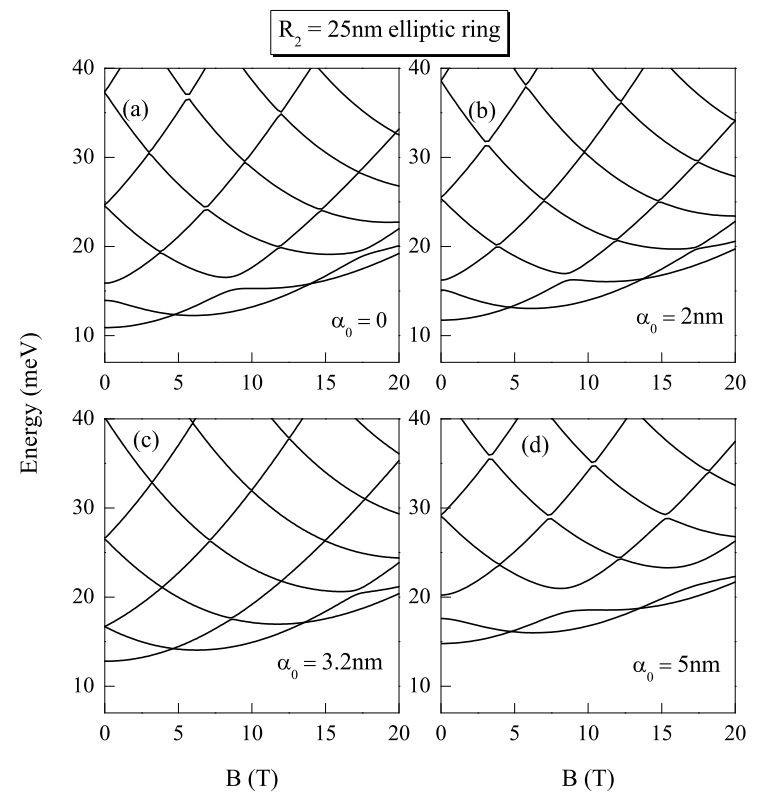

FIG. 3: The low-lying energy levels of an elliptic QR as a function of the magnetic field $B$ for different values of $\alpha_{0}$. The results are for $R_{2}=25 \mathrm{~nm}$.

of the magnetic field $B$ for various values of the laser field parameter $\alpha_{0}$. In Fig. 2 (a) the regular $\mathrm{AB}$ effect has been observed without the laser field, which corresponds to the case of a circular QR. The ILF applied on a QR creates an anisotropy in the confinement potential [Fig. 1 (b)] as a result of which the effective length of the confinement along the $x$-direction decreases in the lower part of QR potential well. It is worth noting that with the increase of $\alpha_{0}$, the anisotropy of the QR is strengthened and the degeneracy of the excited states at $B=0$ disappears. With an increase of $\alpha_{0}$ due to the reducing symmetry from $C_{\infty}$ to $C_{2}$, one should expect an energy spectrum split into non-crossing pairs of states which in turn cross repeatedly as $B$ increases (each pair of repeatedly crossing states containing one instance of each of two $C_{2}$ symmetries). A similar behavior of the energy levels, which can be called 'unusual' or 'irregular' $\mathrm{AB}$ oscillations, have been reported earlier in QRs by other authors that is caused by the effective mass anisotropy [24, 26] and structural distortions in QRs [22]. For $\alpha_{0}=2$ $\mathrm{nm}$, only the ground and first excited states feel the deformation of the potential (see Fig. 2(b)). Whereas, for larger values of $\alpha_{0}$ more excited states start to feel the deformation of the QR confinement potential and the two periodically crossing pairs can be visible [Fig.2 (d)].

Let us now consider the case of the anisotropic QR under the action of ILF. From Eq. (2) and Fig.1(c) it is clear that if $\varepsilon \neq 0$ the undressed confinement potential is anisotropic, and the QR is compressed along the $y$ direction. On the other hand the laser field brings

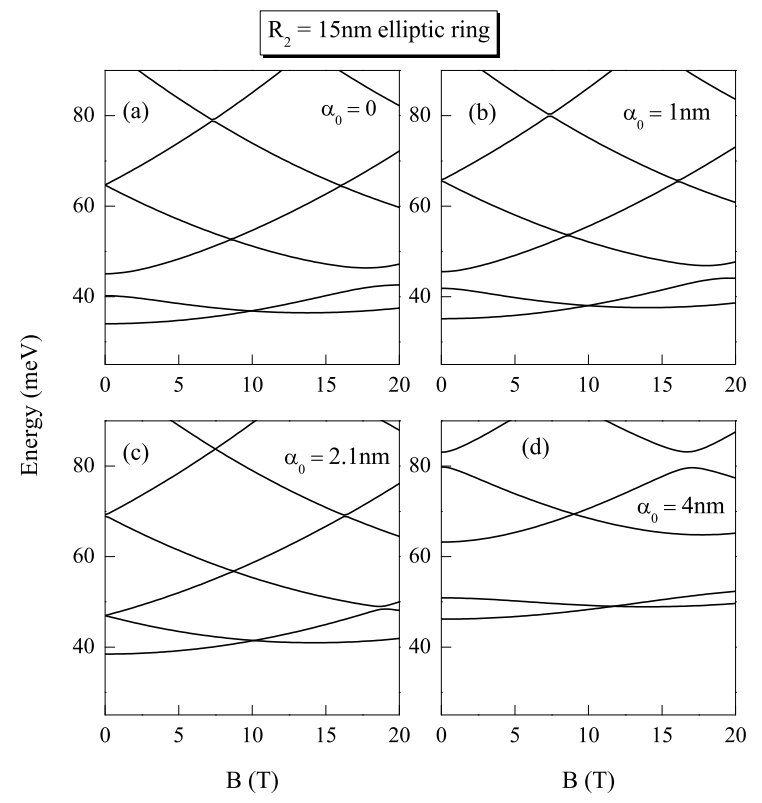

FIG. 4: The low-lying energy levels of an elliptic QR as a function of the magnetic field $B$ for different values of $\alpha_{0}$. The results are for $R_{2}=15 \mathrm{~nm}$.

an anisotropy of the confinement potential along the $x$ direction. Therefore, we have two competing different effects, the first one is caused by the structural anisotropy of the system while the other is caused by the external ILF. In Fig. 3 the magnetic field dependence of the lowlying energy levels are presented, for an anisotropic QR $(\varepsilon=0.5)$ and for different values of $\alpha_{0}$. Fig. 3(a) displays the irregular $\mathrm{AB}$ oscillations without the ILF due to the structural anisotropy of the QR. With an increase of $\alpha_{0}$ the effect of structural anisotropy on the energy levels weakens (Fig. 3(b)), and for $\alpha_{0}=3.2 \mathrm{~nm}$ the regular $\mathrm{AB}$ oscillations are completely recovered (Fig. 3(c)). A farther increase of the ILF parameter again creates an anisotropy in the $x$ direction, and again the irregular $\mathrm{AB}$ oscillations can be observed in Fig. 3(d). This reentrant behavior of the electronic states have never been reported earlier in a quantum ring. Similar effects also can be observed for smaller QRs, which is presented in Fig. 4. The influence of ILF on the confinement potential of the QR is stronger for smaller $\mathrm{QRs}$, and therefore the regular $\mathrm{AB}$ oscillations are recovered for $\alpha_{0}=2.1 \mathrm{~nm}$ (see Fig. 4(c)).

These interesting properties of the energy spectra are expected to influence the optical properties of the QRs. In Fig. 5 the dipole-allowed optical transition energies as a function of the magnetic field are presented for different values of $\alpha_{0}$ for isotropic $\mathrm{QR}$ with outer radius $R_{2}=25$ $\mathrm{nm}$. The size and the color of the circles in this figure is proportional to the intensity of the calculated optical transitions. Without the laser field the signature of the usual AB optical oscillations is seen in Fig. 5(a). The 


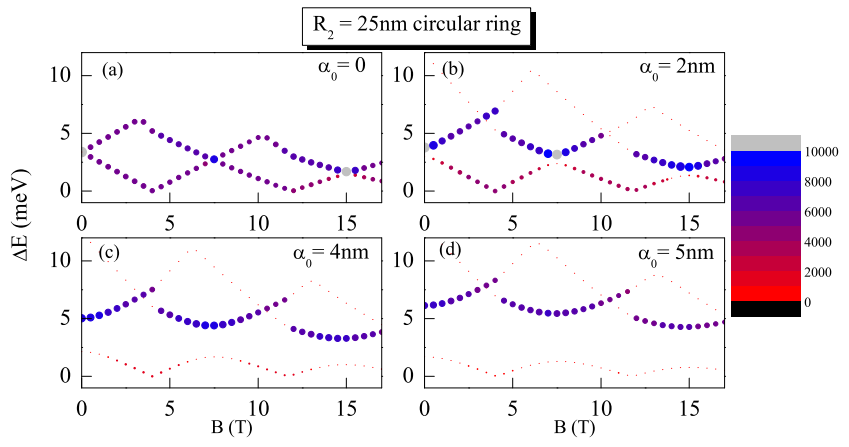

FIG. 5: Dipole allowed optical transition energies as a function of magnetic field $B$ for different values of $\alpha_{0}$. The results are for a $R_{2}=25 \mathrm{~nm}$ circular QR. The size and the color of the circles is proportional to the intensity of the calculated optical transitions.

energies in Fig. 5(a) correspond to the transitions from the ground state to the first and second excited states. All other transitions are forbidden due to the cylindrical symmetry of the structure. With the increase of $\alpha_{0}$ the irregular optical $\mathrm{AB}$ oscillations are again visible. Further, it should be noted that with the increase of $\alpha_{0}$ the intensity of the $1 \rightarrow 2$ transition weakens and the intensity of the $1 \rightarrow 3$ transition strengthens. As an example, for $\alpha_{0}=5 \mathrm{~nm}$ the $1 \rightarrow 2$ transition has almost disappeared. This fact can be explained by the anisotropy of the system created by the ILF in the $x$ direction.

In Fig. 6 the same results as in Fig. 5 are presented for an anisotropic QR and for the value $\varepsilon=0.5$. Without the laser field the optical $\mathrm{AB}$ oscillations again have irregular behavior [Fig. 6(a)], but now the intensity of the $1 \rightarrow 2$ transition is stronger than that of $1 \rightarrow 3$. This is because the structural anisotropy is created in the $y$ direction. With an increase of $\alpha_{0}$ the intensity of $1 \rightarrow 3$ increases and the intensity of $1 \rightarrow 2$ decreases. For the value of $\alpha_{0}=3.2 \mathrm{~nm}$ the regular optical AB oscillations are completely recovered for an anisotropic QR (Fig. 6(c)). Therefore we believe that these interesting effects can be confirmed experimentally.

In conclusion, we have studied here the strong influ- ence of intense terahertz laser field on the electronic and optical properties of isotropic and anisotropic QRs in an applied magnetic field. We have shown that in isotropic QRs the laser field creates the irregular AB oscillations, which is usually expected in anisotropic rings. Therefore with the laser field we can observe a continues evolution of $\mathrm{AB}$ oscillations within the same ring. In the case of anisotropic QRs we have shown that with the ILF it is possible to completely 'regularize' the anisotropy of the $\mathrm{QR}$ and thus also the physical characteristics. In particular we have shown here that energy spectra and $A B$ oscillations have been made completely regular by the ILF for anisotropic QRs. Lastly, it is worth noting that

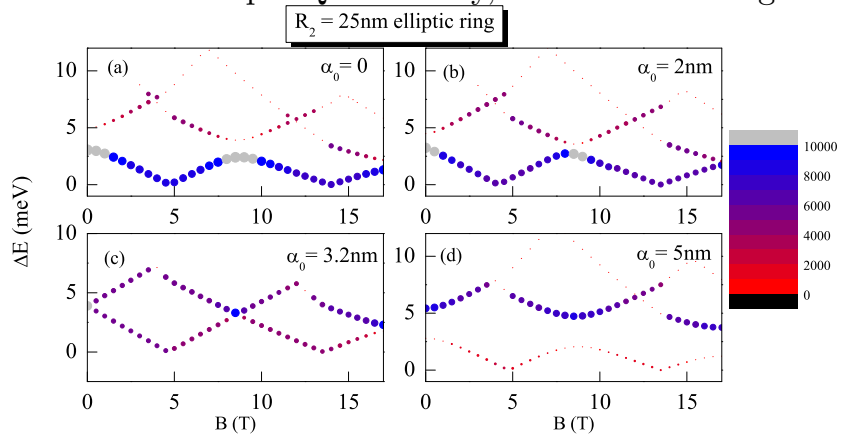

FIG. 6: Dipole-allowed optical transition energies as a finction of the magnetic field $B$ for different values of the laser field parameter $\alpha_{0}$. The results are for $R_{2}=25 \mathrm{~nm}$ elliptic QR. The size and the color of the circles in the figure are proportional to the intensity of the calculated optical transitions.

the ILF can in principle restore isotropic properties of a QR from any type of anisotropy (structural, effective masses, defects, etc.) of the QRs. We believe that in addition to providing an unified picture of the electronic and optical properties of quantum rings, our studies will also open up new possibilities to design, fabricate and improve new devices based on QRs, such as therahertz detectors, efficient solar cells, photon emitters, etc.

The work has been supported by the Canada Research Chairs Program of the Government of Canada, the Armenian State Committee of Science (Project no. 15T1C331), CONICYT-ANILLO ACT 1410, and the center of excellence with BASAL/CONICYT financing, Grant No. FB0807, CEDENNA.
[1] Y. Aharonov, D. Bohm, Phys. Rev. 115, 485 (1959).

[2] M. Büttiker, Y. Imry, R. Landauer, Phys. Lett. A 96, 365 (1983).

[3] A. Lorke, et. al, Phys. Rev. Lett. 84, 2223 (2000).

[4] U.F. Keyser, C. Fühner, S. Borck, R.J. Haug, M. Bichler, G. Abstreiter, and W. Wegscheider, Phys. Rev. Lett. 90, 196601 (2003); A. Fuhrer, S. Lüscher T. Ihn, T. Heinzel1, K. Ensslin, W. Wegscheider, and M. Bichler, Nature 413, 822 (2001).

[5] T. Chakraborty, Adv. in Solid State Phys. 43,
79,(2003); S. Viefers, P. Koskinen, P. Singha Deo, M. Manninen, Physica E 21, 1 (2004); P. Pietiläinen and T. Chakraborty, Solid State Commun. 87, 809 (1993); K Niemelä, P Pietiläinen, P Hyvönen, and T Chakraborty, EPL (Europhysics Letters) 36, 533 (1996); T. Chakraborty and P. Pietiläinen, Phys. Rev. B 50, 8460 (1994).

[6] G. Huang, W. Guo, P. Bhattacharya, G. Ariyawansa, A.G.U. Perera, Appl. Phys. Lett. 94, 101115 (2009).

[7] J. Wu, Z.M. Wang, V.G. Dorogan, S. Li, Z. Zhou, H. Li, 
J. Lee, E.S. Kim, Y.I. Mazur, G.J. Salamo, Appl. Phys. Lett. 101, 043904 (2012).

[8] R.J. Young, E.P. Smakman, A.M. Sanchez, P. Hodgson, P.M. Koenraad, M. Hayne, Appl. Phys. Lett. 100, 082104 (2012).

[9] R.J. Warburton, C. Schäflein, D. Haft, F. Bickel, A. Lorke, K. Karrai, J.M. Garcia, W. Schoenfeld, P.M. Petroff, Nature 405, 926 (2000).

[10] M. Abbarchi, C. Mastrandrea, A. Vinattieri, S. Sanguinetti, T. Mano, T. Kuroda, N. Koguchi, K. Sakoda, M. Gurioli, Phys. Rev. B 79, 085308 (2009).

[11] D.S.L. Abergel, V. Apalkov, J. Berashevich, K. Ziegler, and T. Chakraborty, Adv. Phys. 59, 261 (2010); H. Aoki and M.S. Dresselhaus (Eds.), Physics of Graphene (Springer, New York 2014); J. Berashevich and T. Chakraborty, Nanotechnology 21, 355201 (2010).

[12] A. Tsukazaki, A. Ohtomo, T. Kita, Y. Ohno, H. Ohno, and M. Kawasaki, Science 315, 1388 (2007); A. Tsukazaki, S. Akasaka, K. Nakahara, Y. Ohno, H. Ohno, D. Maryenko, A. Ohtomo and M. Kawasaki, Nature Materials 9, 889 (2010); J. Falson, D. Maryenko, B. Friess, D. Zhang, Y. Kozuka, A. Tsukazaki, J.H. Smet and M. Kawasaki, Nature Physics 11, 347 (2015).

[13] D. Abergel, V. Apalkov, and T. Chakraborty, Phys. Rev. B 78, 193405 (2008); M. Zarenia, B. Partoens, T. Chakraborty and F.M. Peeters, ibid 88, 245432 (2013).

[14] T. Chakraborty, A. Manaselyan and M. Barseghyan, J. Phys.: Condens. Matt. 29, 215301 (2017).

[15] Physics of Quantum Rings, edited by V.M. Fomin (Springer-Verlag, Berlin Heidelberg; 2014).

[16] R. Blossey and A. Lorke, Phys. Rev. E 65, 021603 (2002).

[17] P. Offermans, P.M. Koenraad, J.H. Wolter, D. Granados, J.M. Garcia, V.M. Fomin, V.N. Gladilin and J.T. Devreese, Appl. Phys. Lett. 87, 131902 (2005).

[18] N. A. J. M. Kleemans, I. M. A. Bominaar-Silkens, V. M. Fomin, V. N. Gladilin, D. Granados, A. G. Taboada, J. M. García, P. Offermans, U. Zeitler, P. C. M. Christianen, J. C. Maan, J. T. Devreese, and P. M. Koenraad,
Phys. Rev. Lett. 99, 146808 (2007).

[19] J. Wu, Z.M. Wang, K. Holmes, E. Marega Jr., Z. Zhou, H. Li, Y.I. Mazur, G.J. Salamo, Appl. Phys. Lett. 100, 203117 (2012).

[20] T. Raz, D. Ritter and G. Bahir, Appl. Phys. Lett. 82, 1706 (2003).

[21] J. Sormunen, J. Riikonen, M. Mattila, J. Tiilikainen, M. Sopanen and H. Lipsanen, Nano. Lett. 5, 1541 (2005).

[22] J. Planeles, F. Rajadell, J.I. Climente, Nanotechnology 18, 375402 (2007).

[23] G.A. Farias, M.H. Degani, J.A.K. Freire, J. Costa e Silva, R. Ferreira, Phys. Rev. B 77, 085316 (2008).

[24] M.M. Milošević, M. Tadić, F.M. Peeters, Nanotechnology 19,455401 (2008).

[25] A. Ghazaryan, A. Manaselyan, T. Chakraborty, Phys. Rev. B 93, 245108 (2016).

[26] G.O. de Sousa, D.R. da Costa, Andrey Chaves, G.A. Farais, F.M. Peeters, Phys. Rev. B 95 (2017) 205414.

[27] W. C. Henneberger, Phys. Rev. Lett. 21, 838 (1968).

[28] M. Gavrila, J. Z. Kaminski, Phys. Rev. Lett. 52, 613 (1984).

[29] M. Pont, N.R. Walet, M. Gavrila C.W. McCurdy, Phys. Rev. Lett. 61939 (1988).

[30] E. C. Valadares, Phys. Rev. B(R) 41, 1282 (1990).

[31] M. Gavrila, J. Phys. B: At. Mol. Opt. Phys. 35, R147 (2002).

[32] S.D. Ganichev, W. Prettl (eds.), Intense Terahertz Excitation of Semiconductors. Semiconductor Science and Technology (Oxford University Press, 2006).

[33] A. Radu, A.A. Kirakosyan, D. Laroze, H.M. Baghramyan, M.G. Barseghyan, J. Appl. Phys. 116, 093101 (2014).

[34] A. Radu, A.A. Kirakosyan, D. Laroze, M.G. Barseghyan, Semicond. Sci. Technol. 30, 045006 (2015).

[35] S. Adachi, Handbook on Physical Properties of Semiconductors. v. 2, Kluwer Academic Publishers, New York 2004 . 